\title{
Investigating the past history of EXors: the cases of V1118 Orionis, V1143 Orionis, and NY Orionis *
}

\author{
R. Jurdana-Šepić ${ }^{1}$, U. Munari ${ }^{2}$, S. Antoniucci ${ }^{3}$, T. Giannini ${ }^{3}$, G. Li Causi ${ }^{3,4}$, and D. Lorenzetti ${ }^{3}$ \\ 1 Physics Department, University of Rijeka, Radmile Matejčić, 51000 Rijeka, Croatia \\ 2 INAF-Osservatorio Astronomico di Padova, via dell'Osservatorio 8, 36012 Asiago (VI), Italy \\ 3 INAF-Osservatorio Astronomico di Roma, via Frascati 33, 00078 Monte Porzio Catone, Italy \\ e-mail: antoniucci@oa-roma.inaf.it \\ ${ }^{4}$ INAF-Istituto di Astrofisica e Planetologia Spaziali, via Fosso del Cavaliere 100, 00133 Roma, Italy
}

Received 21 December 2016 / Accepted 23 February 2017

\begin{abstract}
Context. EXor objects are young variables that show episodic variations of brightness commonly associated to enhanced accretion outbursts.

Aims. With the aim of investigating the long-term photometric behaviour of a few EXor sources, we present here data from the archival plates of the Asiago observatory, showing the Orion field where the three EXors V1118, V1143, and NY are located.

Methods. A total of 484 plates were investigated, providing a total of more than 1000 mag for the three stars, which cover a period of about 35 yr between 1959 to 1993 . We then compared our data with literature data.

Results. Apart from a newly discovered flare-up of V1118, we identify the same outbursts already known, but we provide two added values: (i) a long-term sampling of the quiescence phase; and (ii) repeated multi-colour observations (BVRI bands). The former allows us to give a reliable characterisation of the quiescence, which represents a unique reference for studies that will analyze future outbursts and the physical changes induced by these events. The latter is useful for confirming whether the intermittent increases of brightness are accretion-driven (as in the case of V1118), or extinction-driven (as in the case of V1143). Accordingly, doubts arise about the V1143 classification as a pure EXor object. Finally, although our plates do not separate NY Ori and the star very close to it, they indicate that this EXor did not undergo any major outbursts during our $40 \mathrm{yr}$ of monitoring.
\end{abstract}

Key words. stars: pre-main sequence - stars: variables: T Tauri, Herbig Ae/Be - catalogs - stars: individual: NY Ori stars: individual: V1118 Ori - stars: individual: V1143 Ori

\section{Introduction}

Among the low-mass protostars, the EXor-type objects (hereafter EXors - Herbig 1989) are considered a peculiar sub-class of the classical T Tauri stars (CTTS). These sources undergo episodic outbursts of brightness (typically of three to five magnitudes at optical wavelengths) caused by sudden variations of the mass accretion rate that obeys the magnetospheric accretion rules (Shu et al. 1994). At the moment, the known EXors are only few tens (Audard et al. 2014) usually discovered by chance. Indeed, all the long term photometric surveys carried out so far on CTTS samples confirm that they present, on average, small amplitude variations (typically $\lesssim 1 \mathrm{mag}$ ). These results tend to support a certain peculiarity of the EXors, but to what extent their accretion events are unfrequent manifestations of a common phenomenology (Lorenzetti et al. 2012) is not firmly ascertained.

EXors present short outburst (months-one year) with a recurrence time of years, associated with accretion rates of the order of $10^{-6}-10^{-7} M_{\odot} \mathrm{yr}^{-1}$, and characterised by emission-line spectra (e.g. Herbig 2008; Lorenzetti et al. 2009; Kóspál et al. 2011; Sicilia-Aguilar et al. 2012; Antoniucci et al. 2013, 2014). Such

\footnotetext{
* Full Table 1 is only available at the CDS via anonymous ftp to cdsarc.u-strasbg. fr (130.79.128.5) or via http://cdsarc.u-strasbg.fr/viz-bin/qcat?J/A+A/602/A99
}

features, and in particular the burst cadence, make EXors the ideal candidates for a long-term monitoring, which should be effective in comparing the properties of several subsequent events and evidence their similarities or differences. Following this observational approach, compelling constraints can be provided for the models currently available. Indeed, the EXors phenomenology has been so far interpreted by borrowing the theoretical approaches developed to study the FUor events (Hartmann \& Kenyon 1985), but a detailed model of the disk structure and its evolution does not exist yet for EXors stars. D'Angelo \& Spruit (2010) provided quantitative predictions for the episodic accretion of piled-up material at the inner edge of the disk that, however, are largely incompatible with the observations. Hence, the mechanism responsible for the onset of EXor accretion outbursts remains unknown to date. Two proposed scenarios involve essentially either disk instabilities (gravitational - e.g. Adams \& Lin 1993; or thermal - e.g. Bell \& Lin 1994) or perturbation by an external body (a close encounter in a binary system - e.g. Bonnell \& Bastien 1992; or presence of a massive planet - e.g. Lodato \& Clarke 2004).

The young star V1118 Ori, one of the classical EXor objects, represents a very suitable target for such a kind of long-term monitoring. During its recent history V1118 Ori underwent six documented outbursts, each lasting a couple of years (1982-84, 1988-90, 1992-94, 1997-98, 2004-06, 
2015-on going). Account for the first five events is given in Parsamian et al. (1993), García García \& Parsamian (2000), Herbig (2008) and references therein, Audard et al. (2005, 2010), and Lorenzetti et al. (2006, 2007). The properties shown during the long quiescence period before the last eruption, firstly detected around Sept. 2015, are described in Lorenzetti et al. (2015), Giannini et al. (2016, and in prep.). In this latter paper, a comparison of all the recent outbursts is presented, with the aim of investigating if any periodicity is recognizable among the recurrent events. However, so far only a couple of outbursts have been sampled at an accuracy level that allows us to have some indication on this aspect. A firm ascertainment of the existence of a periodicity could speak in favour of a disk instability generated by an external body as the most likely mechanism for the mass accretion variations. To this end, we have investigated the optical plates collected by the Asiago (Italy) Schmidt telescopes during a timespan of about 40 yr (1958-1998), which are able to provide the most accurate long-term light curve of V1118 Ori available before the advent of both CCD and near-IR arrays.

Beside V1118 Ori, two other EXors, namely V1143 Ori and NY Ori, are also located in the Orion Nebula Cluster (ONC), thus appearing in the same archival plates of the Asiago observatory. By analysing these large number of plates we have therefore the great advantage of investigating the past history of two additional systems with the same method and accuracy. V1143 is a very active variable (see Herbig 2008, and references therein) and presents ranges of photometric fluctuations of $\sim 19-14.3 \mathrm{mag}$ and 16.7-13.5 mag in $B / p g$ and $V / v s$ bands, respectively. NY Ori (= Parenago 2119) is a young star located 5.5 arcsec SE of the much optically brighter source V566 Ori (= Parenago 2118). Its photometric history and variability is documented (Herbig 2008) by a series of plates collected during short periods $(1905-19 ; 1948-49 ; 1973)$ with very sparse and occasional sampling, which provide a range of variability in the visual band between 13.3 and 16 mag. A previous plate analysis of V1118 Ori and V1143 Ori was presented by Paul et al. (1995), but the extremely low number of resulting photometric data points (less than ten in 40 years), hampers a proper time coverage.

In any case, the relevance of the plates analysis for studying the eruptive variables is irrefutable, as recently demonstrated for the EXor GM Cep (Xiao et al. 2010) and the FUor V960 Mon (Jurdana-Šepić \& Munari, 2016). Noticeably, the chance of studying the historical light curves of EXors located in the Orion nebula is offered uniquely by the Asiago plates. Indeed, other remarkable archives (e.g. Harvard and Sonneberg) present some difficulties in this respect: firstly, they are not as much deep to investigate the quiescence phase; secondly, they cover almost exclusively the $B$ or blue-unfiltered bands; thirdly, they have been obtained with short focal length astrographs that typically squeeze the plate scale making indistinguishable the stars inside the nebula. To our best knowledge, plate-based monitoring of other EXors as faint as V1118 Ori is not available in literature. The features mentioned above suggest that the Asiago archive might be a reference source for investigating the past history of both recently discovered EXors and those that will be discovered in the next future.

The present paper is organized as follows: the adopted method and the obtained $B V R I$ photometry are presented in Sects. 2 and 3. Section 4 gives the analysis and discussion of the obtained results, while our concluding remarks are given in Sect. 5 .

\section{Data acquisition}

\subsection{Archive plates}

Two Schmidt telescopes are operated at the Asiago observatory. The smaller one (SP: $40 / 50 \mathrm{~cm}, 100 \mathrm{~cm}$ focal length) collected 20417 plates between 1958 to 1992 (covering a circular area $5^{\circ}$ in diameter), and the larger one (SG: $67 / 92 \mathrm{~cm}, 208 \mathrm{~cm}$ focal length) 18811 plates from 1965 to 1998 (imaging a $5^{\circ} \times 5^{\circ}$ portion of the sky). The Asiago Schmidt plate collection thus span $40 \mathrm{yr}$, covering in particular the Menzel Gap during which acquisition of photographic plates was temporarily halted at Harvard Observatory. Since the 1990s, large format CCD cameras have replaced photographic plates as detectors, with the plates preserved in controlled conditions.

The Asiago Schmidt plates have not been exposed over the whole sky but instead on selected targets (in particular star forming regions, clusters of galaxies, galaxies of the Local Group), for which therefore exist a long record of observations. The Orion nebula - around which our targets are located - has been a favoured hit. Nearly all plates from both telescopes go very deep, $B \sim 18.5$ and $B \sim 17.8$ mag being the typical limiting magnitude for blue sensitive plates exposed with the SG and SP telescope, respectively.

The majority of the plates were exposed as 103a-O + GG13, 103a-E + RG1 and IN + RG5 combinations of Kodak plates and Schott astronomical filters, matching the prescription for the Johnson-Cousins $B, R_{\mathrm{C}}$ and $I_{\mathrm{C}}$ photometric bands (Moro $\&$ Munari 2000), respectively. A large number of plates were exposed as unfiltered 103a-O, thus covering both the Johnson $B$ and $U$ bands thanks to the high ultraviolet transparency of the UBK-7 corrector plates at both Schmidt telescopes. For low temperature and/or reddened objects, especially if they were observed at large airmass, the amount of proper $U$-band photons collected by an unfiltered $103 \mathrm{a}-\mathrm{O}$ plate is however minimal compared to those arriving through the $B$-band portion of the interval of sensitivity of 103a-O emulsion. In such conditions (and provided that the selected comparison stars are themselves of low temperature and/or high reddening), 103a-O + GG13 pairs and unfiltered 103a-O plates are almost equally well replicating the standard Johnson $B$ band (Munari \& Dallaporta 2014).

The plates in the proper Johnson $V$ band obtained as 103 a-D + GG14 combination, are low in number, and equally infrequent are unfiltered panchromatic plates as $103 \mathrm{a}-\mathrm{G}, 103 \mathrm{a}-$ $\mathrm{D}$, Tri-X and Pan-Roy. In the present paper, these unfiltered panchromatic plates have been treated as $V$-band plates (even if they lack the coupling to a high-pass filter such as GG11 or GG14) because of the low temperature and reddening of our targets and generally the high airmass $(\sim 1.8)$ of the corresponding observations which prevent $U$ - and $B$-band photons to be collected in any significant number by unfiltered panchromatic plates.

\subsection{Brightness measurement}

To derive the brightness of our targets, we compared them at a high quality Zeiss microscope against a local photometric sequence established around each target. Such a sequence, composed of stars of roughly the same colour as the variable and widely distributed in magnitude so to cover both quiescence and outburst states, was extracted primarily from the APASS $B V g^{\prime} r^{\prime} i^{\prime}$ all-sky survey (Henden et al. 2012; Henden \& Munari 2014), with porting to Landolt $R_{\mathrm{C}}$ and $I_{\mathrm{C}}$ bands following Munari (2012) and Munari et al. (2014) prescriptions. To evaluate the measurement errors a number of plates were re-measured 
R. Jurdana-Šepić et al.: Past history of EXors

Table 1. BVRI plate photometry of NY, V1143, V1118 in Orion.

\begin{tabular}{|c|c|c|c|c|c|c|c|c|c|c|c|c|c|c|c|}
\hline \multirow[b]{2}{*}{ HJD } & \multirow[b]{2}{*}{ Date } & \multirow[b]{2}{*}{ UT } & \multirow[b]{2}{*}{ Emulsion } & \multirow[b]{2}{*}{ Filter } & \multirow[b]{2}{*}{ Tel } & \multirow[b]{2}{*}{ Plate } & \multicolumn{3}{|c|}{ NY Ori } & \multicolumn{3}{|c|}{ V1143 Ori } & \multicolumn{3}{|c|}{ V1118 Ori } \\
\hline & & & & & & & Band & Mag & Error & Band & Mag & Error & Band & Mag & Error \\
\hline 2436545.43698 & $1958-12-07$ & $22: 22$ & 103 a-E & RG 1 & SP & 59 & $R$ & 10.12 & 0.10 & $R$ & 15.56 & 0.10 & $R$ & 15.13 & 0.20 \\
\hline 2436567.31360 & $1958-12-29$ & $19: 25$ & $103 \mathrm{a}-\mathrm{O}$ & $\ldots$ & SP & 66 & $B$ & 10.97 & 0.10 & $B$ & $>15.86$ & 0.10 & $B$ & $*$ & \\
\hline 2436570.29404 & 1959-01-01 & $18: 57$ & $103 \mathrm{a}-\mathrm{O}$ & $\ldots$ & SP & 70 & $B$ & 10.83 & 0.10 & $B$ & $>15.86$ & 0.10 & $B$ & $*$ & \\
\hline 2436570.36626 & 1959-01-01 & $20: 41$ & 103 a-E & RG 1 & SP & 73 & $R$ & 10.12 & 0.10 & $R$ & $>14.74$ & 0.10 & $R$ & 15.13 & 0.20 \\
\hline 2436572.31340 & 1959-01-03 & $19: 25$ & $103 \mathrm{a}-\mathrm{O}$ & ... & SP & 78 & $B$ & 10.90 & 0.10 & $B$ & $>15.86$ & 0.10 & $B$ & $*$ & \\
\hline 2436573.34808 & 1959-01-04 & $20: 15$ & $103 \mathrm{a}-\mathrm{O}$ & $\ldots$ & SP & 81 & $B$ & 10.90 & 0.10 & $B$ & $>17.15$ & 0.10 & $B$ & $*$ & \\
\hline 2436578.30825 & 1959-01-09 & $19: 18$ & $103 \mathrm{a}-\mathrm{O}$ & $\ldots$ & SP & 92 & $B$ & 10.90 & 0.10 & $B$ & $>17.15$ & 0.10 & $B$ & $*$ & \\
\hline 2436579.32278 & 1959-01-10 & $19: 39$ & $103 \mathrm{a}-\mathrm{O}$ & $\ldots$ & SP & 99 & $B$ & 10.90 & 0.10 & $B$ & $>17.15$ & 0.10 & $B$ & $*$ & \\
\hline 2436581.30115 & $1959-01-12$ & $19: 08$ & $103 \mathrm{a}-\mathrm{O}$ & $\ldots$ & SP & 113 & $B$ & 10.90 & 0.10 & $B$ & $>17.15$ & 0.10 & $B$ & $*$ & \\
\hline 2436582.38858 & 1959-01-13 & $21: 14$ & $103 \mathrm{a}-\mathrm{O}$ & $\ldots$ & SP & 127 & $B$ & 10.90 & 0.10 & $B$ & $>17.15$ & 0.10 & $B$ & $*$ & \\
\hline
\end{tabular}

Notes. Columns provide: date and central UT of any exposure, plate emulsion, the adopted filter, the telescope, the plate number, the band, and the magnitude derived for the three sources with the estimated error (see text for further details). An asterisk indicates cases for which it was only possible to rule out that the source was going through a bright outburst. The full table is available at the CDS.

after several days when all memories had vanished from the observer, and independently by different observers. The typical error is $0.1 \mathrm{mag}$, comparable to that intrinsic to the photographic plate itself so that the observer adds little to it. When a larger error is reported, that is usually associated to presence of a fogged background, a trailed guiding, or poor seeing.

A total of 484 plates imaging the Orion nebula were retrieved from the Asiago Schmidt plate archive. After inspection, 44 of them were rejected for various reasons (too short exposures, unsuitable emulsion or filter combinations, damages during development or storing, etc.), leaving a total of 440 plates ready for measurement, spanning the time interval from Dec. 7 , 1958 to Dec. 12, 1993. The results are given in Table 1 where the columns provide date/UT/HJD of observation, photographic emulsions and filters, plate number and telescope, the estimated magnitude or plate limit, and the corresponding errors. When the variable is too faint to be detected, the limiting magnitude is listed as that of the faintest of the stars in the comparison sequence which is clearly visible.

The faint variables V1118 Ori and V1143 Ori are superimposed to the Orion nebula, which greatly disturbs their detection and measurement. All emission from the nebula come from few extremely intense emission lines, mainly [OIII] 4959, $5007 \AA$ and the $[\mathrm{NII}]+\mathrm{H} \alpha$ complex. A minimal variance in the transmission of the emulsion+filter combination at these wavelengths causes large changes in the brightness background. For this reason, in a significant number of plates the nebular background around V1118 Ori is so bright that it is impossible to estimate the brightness in quiescence of such a faint variable star. For these plates (identified in Table 1 and whose date of observation is depicted in Fig. 1) it was only possible to exclude that the target was at that time going through a bright outburst. NY Ori is a member of a close optical pair with brighter 2MASS J05353579-0512205 (= V566 Ori) at 5 arcsec distance. On the Palomar Schmidt plates they can be percept as a heavily blended pair, with the two stars not measurable separately, while at the shorter focal length of the Asiago Schmidt telescopes the pair is merged into the image of a unresolved single star. Therefore the measurements of NY Ori reported in Table 1 refer to the combined pair of stars.

\section{Historical light curves}

The BVRI light curves of V1118, V1143, and NY Ori corresponding to their plate photometry given in Table 1, are given in Figs. 1-3, respectively. In Table 2 some statistics are provided for the three sources in each band, namely the number of observations, the median value (basically the quiescence magnitude), together with the standard deviation of the data points, and the peak brightness. Notably, we provide one of the best sampled data set ever obtained of the quiescent phase of the investigated sources. Indeed, albeit observations are concentrated within the seasonal periods of observability, their number is significantly high (between 50 and 100) in many cases, allowing us to infer meaningful averaged values for the quiescence level. Determining this level is fundamental to have a solid reference for future observations that will analyse new outbursts and investigate the physical changes induced on the system by these enhanced accretion events. Finally, we note how these sources (especially V1118 and V1143) present, in quiescence, a level of modest variability. This may be quantified by considering the standard deviation of the measurements $(0.2-0.4 \mathrm{mag})$, which is comparable with that of the classical T Tauri (CTTS).

\section{Analysis and discussion}

\subsection{V1118 Ori}

Examining its light curve in $R$ and $I$ bands, depicted in Fig. 1, three major ( $\Delta m \gtrsim 2 \mathrm{mag}$ ) flare-up events are recognizable: a first one in 1966 February ( $R$ band), a second one in 1983 November ( $R, I$ bands), and a third one in 1989 February $(B, R$, and $I$ bands). This first event is mentioned here for the first time; however, as indicated in Table 1 (HJD 2439 172), it was a sudden flaring more than an EXor typical outburst, since the $R$ band brightness increased by $2.5 \mathrm{mag}$ in $40 \mathrm{~min}$, representing a very fast onset of a flare lasting less than one months (see $I$ band values in the same table). Similar episodes, usually associated with very active chromospheres and hence detected at higher energies, are not commonly observed in either this source or other EXors, but, unfortunately, this episode has been sampled only in the $R$ band, thus preventing any colour analysis (see below).

However, it apparently marks the beginning of a long quiescence period from 1966 to 1981, as testified by the subsequent monitoring in the $I$ band, whose temporal coverage suggests the absence of potential outbursts lasting more than 1 yr. Two observational gaps, slightly longer than $1 \mathrm{yr}$, indeed exist in 1973-74 and 1975-76 (see Table 1), so we cannot exclude that an outburst might have occurred during one or both these observational gaps. Remarkably, this quiescence period (about $15 \mathrm{yr}$ ) would be the longest known so far (Lorenzetti et al. 2015), thus its relevance is twofold: firstly, it poses a compelling constraint on the existence 


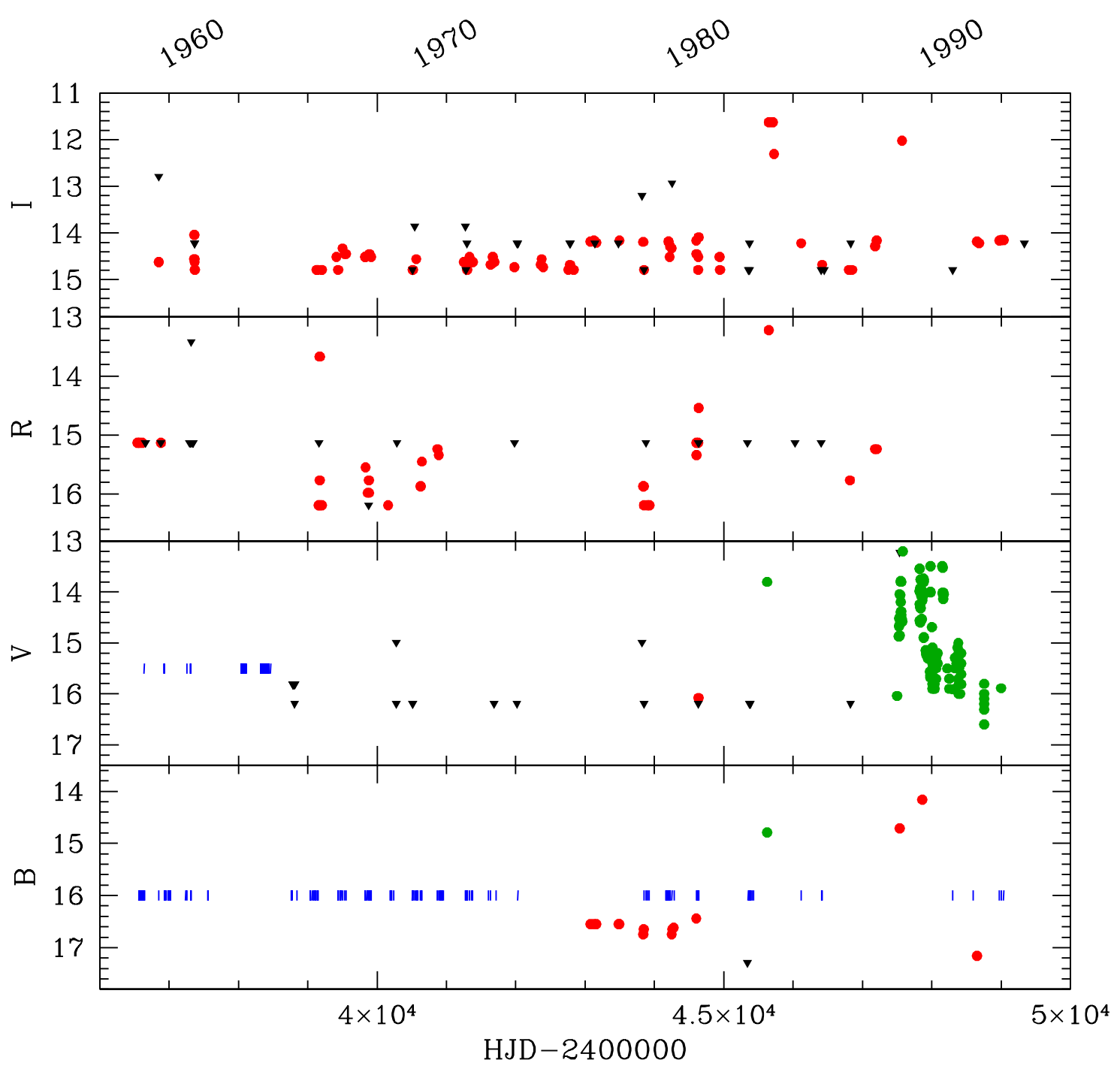

Fig. 1. BVRI light curves of V1118 Ori. Upper limits are given as solid black triangles. In the two bottom panels $(B$ and $V)$, blue vertical bars indicate the dates when the nebular background around V1118 Ori was too bright (see text Sect. 2.2). Green points refer to literature measurements reported by Parsamian et al. (1993) and García García \& Parsamian (2000).

of a recurrent interval between two bursts; secondly, it allows us to define a reliable quiescent value of $I=14.6 \mathrm{mag}$ (see Table 2). The second and third events are already mentioned in the literature by Parsamian et al. (1993), García García \& Parsamian (2000), who gave only the $B$ and $V$ magnitudes: our photometry is in agreement with their values, but provides, for the first time, the $R$ and $I$ magnitudes, as well. In particular for the third event, by considering only the simultaneous plates (i.e. taken at a maximum temporal distance of one day), we are able to build a colour-magnitude plot $B$ vs. $[B-I]$ practically unaffected by short time fluctuations and shown in the left panel of Fig. 4. Still unpublished data (Giannini et al., in prep.), that we collected during the very recent $2015-2016$ outburst (Giannini et al. 2016) are shown on the figure, as are data from the current plate study. In both cases, the source presents the very common behaviour to become bluer when brightening, but definitely not following the extinction law (depicted by the arrow). These colours provide a further confirmation that the increase of brightness of V1118 Ori is accretion- more than extinction-driven (Lorenzetti et al. 2015). Moreover, by considering the two distributions of open and solid data points, one could suspect that the different origin of the two data set (CCD and plates) has a role in determining the colour segregation, but this is not true as confirmed by the green data points relative to the 2005 outburst (Audard et al. 2005). The 2005 data perfectly overlap the plate photometry presented here, thus testifying on the one hand the reliability of the Asiago plates, on the second hand the fact that different bursts may have different colours and intensities.

\subsection{V1143 Ori}

Two bursts are recognizable in our light curves (see Fig. 2 and also Table 1): the first appears as a steep rise up to a maximum in January 1983, followed by a slower declining; the second occurred in January 1993 and presents the same rapid increase (less than one month). Mention of both events was already given by Parsamian \& Gasparian (1987) and Mampaso \& Parsamian (1995), respectively, but quiescence data in the same bands are not provided, so preventing any comparative colour analysis. Our plate data, instead, allow this comparison, given in the right panel of Fig. 4. This latter indicates the two bursts were practically identical in both $B$ amplitude and $[B-I]$ colour, and, more importantly, that this two bursts follow exactly the extinction 


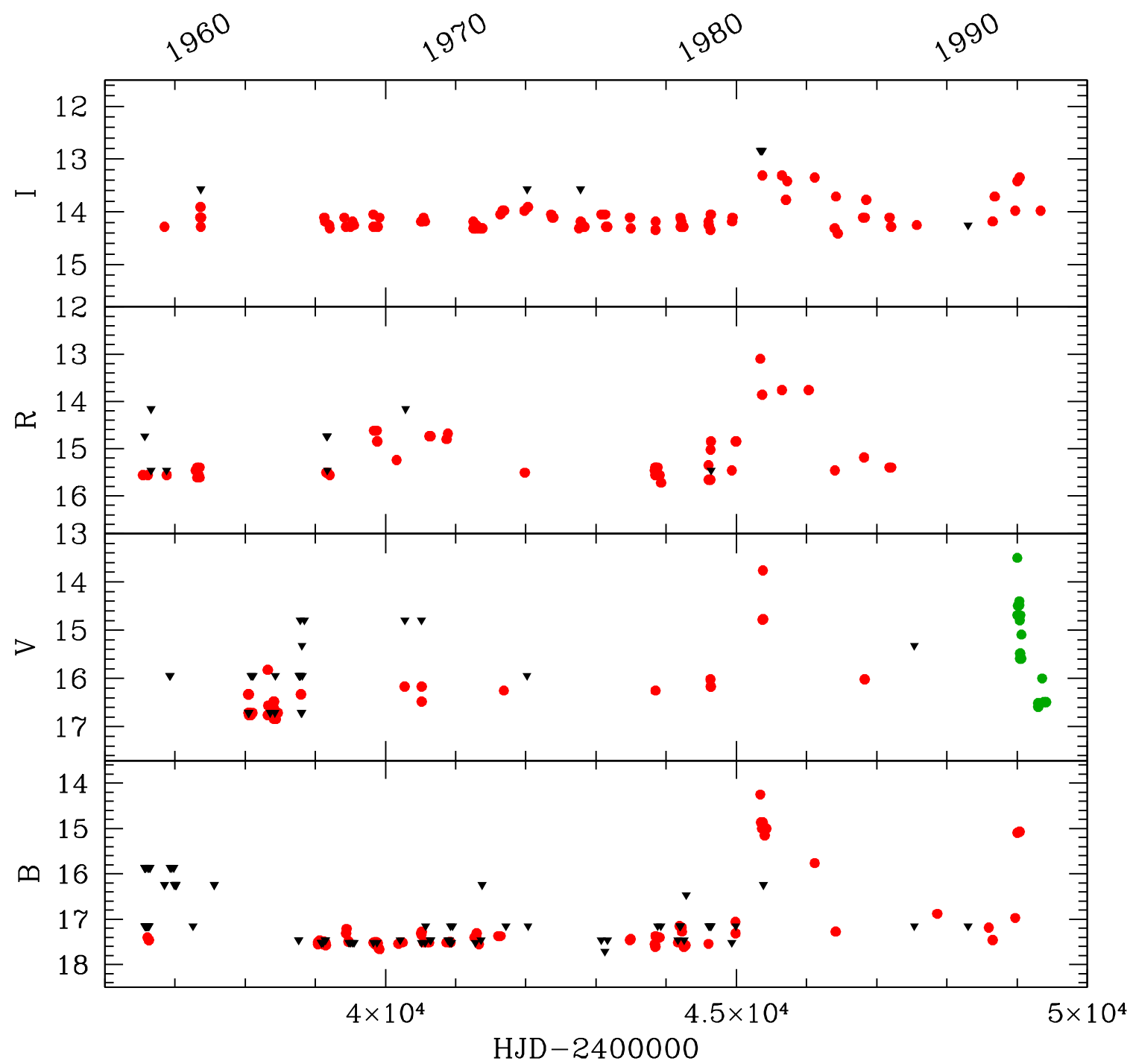

Fig. 2. BVRI light curves of V1143 Ori. Upper limits are given as solid black triangles. Green points are measurements taken from by Mampaso \& Parsamian (1995).

curve with a variation corresponding to $\Delta A_{V}=2$ mag. This result, if confirmed by future observations, could indicate V1143 Ori is not a genuine EXor, since both its historical outbursts are severely contaminated by extinction effects. As for V1118, our data provide a good overall sampling of the quiescent phase, in fact the $I$-band photometry fills the temporal gap 1972-1977 uncovered in the $B V R$ bands. Our quiescence photometry agrees with the sparse values listed so far in the literature (Herbig 2008, and references therein).

\subsection{NY Ori}

As previously mentioned, the measurements of NY Ori (Table 1) actually refer to the combination of the EXor source and the close star V566 Ori. Optical light curves (Fig. 3) seem dominated by the brighter V566 Ori in the bands $B V R$, while in the $I$ band the EXor becomes to prevail. The shortest wavelength colours are compatible with an early type star (SpT A-F), whereas at longer wavelength colours become redder and are those typical of a late type source. A confirmation of that stems from the $J H K$ near-infrared photometry carried out at our telescope at Campo Imperatore (D'Alessio et al. 2000). For V566 Ori, we obtained $J=9.73, H=9.70$, and $K=9.70$, in agreement with 2MASS results $(J=9.75, H=9.74$, and $K=9.73)$; while our IR monitoring of the EXor NY Ori provided evidence of both redder colours and some variability: $J(9.8-10.6)$, $H$ (8.9-9.4), and $K(8.1-8.5)$. At the same telescope, we obtained low-resolution $(\mathcal{R} \sim 200)$ near-IR spectra $(1-2.5 \mu \mathrm{m})$ of both sources. V566 Ori is characterised by strong HI absorption lines (Paschen and Brakett series), while the EXor appears as a typical emission-line object (Lorenzetti et al. 2009). Finally, we note in Fig. 3 how the temporal coverage in $V$ band fills the gap of $I$ band observations in the period 1961-1967, so we can conclude that during our $40 \mathrm{yr}$ monitoring no major outburst occurred with a duration longer than six months. Such a conclusion is based on the analysis of the $I$ ligthcurve, namely that in which both stars have a comparable brightness.

\section{Concluding remarks}

Archival plate analysis is a powerful tool to investigate the historical behaviour of EXor stars, and to infer on their nature.

We investigated the Asiago Schmidt plate collection for observations of the Orion Nebula Cluster where the three EXors 


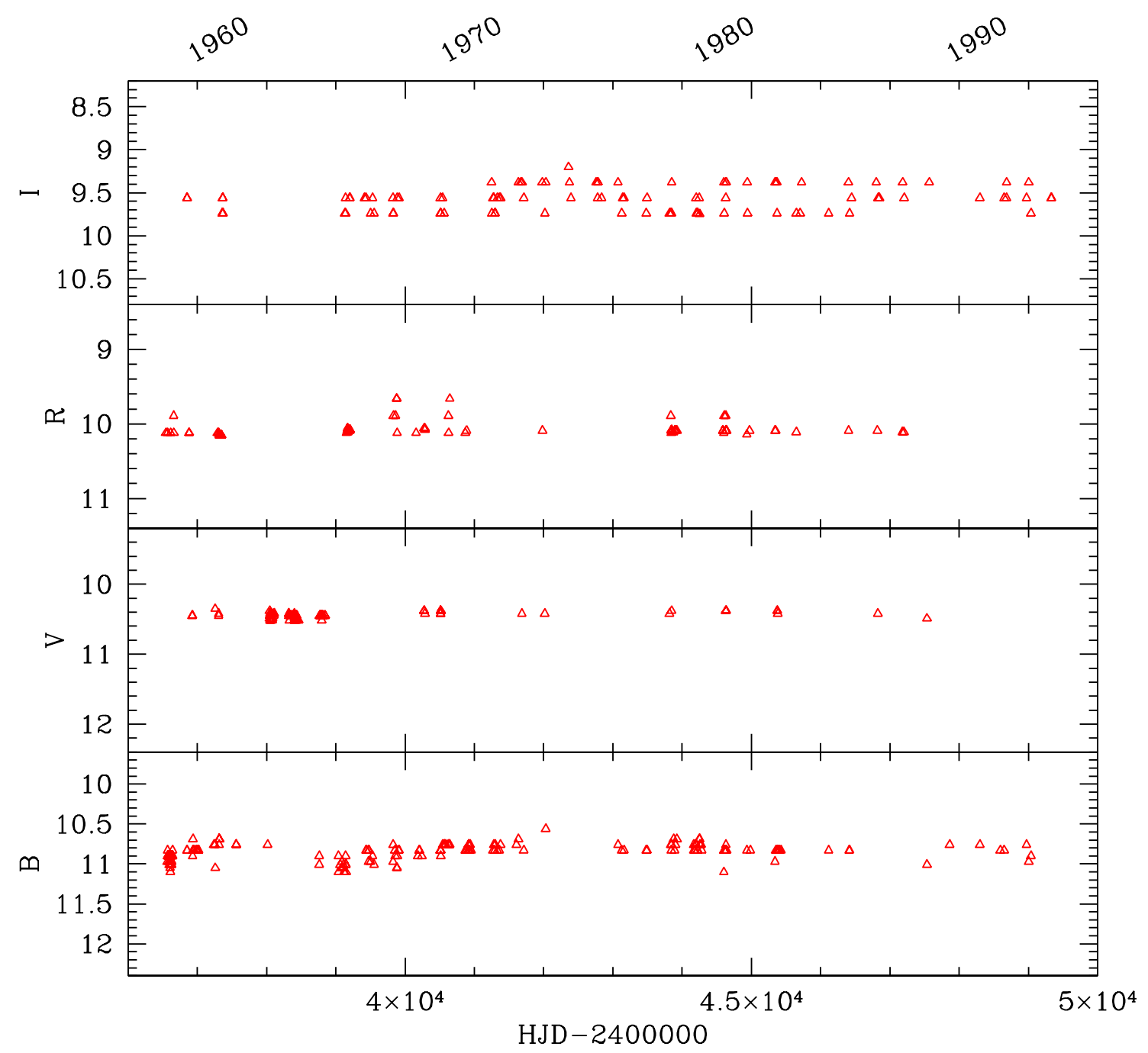

Fig. 3. BVRI light curves of NY Ori.

Table 2. Ranges of photometric variability.

\begin{tabular}{lcc|ccc}
\hline \hline Source & Band & $N_{\text {obs }}$ & Median & $\begin{array}{c}\sigma \\
(\mathrm{mag})\end{array}$ & Peak \\
\hline V1118 Ori & $B$ & 13 & 16.62 & 0.25 & 14.16 \\
& $V$ & 2 & 16.02 & $\ldots$ & $\ldots$ \\
& $R$ & 33 & 15.51 & 0.64 & 13.33 \\
& $I$ & 69 & 14.56 & 0.24 & 11.63 \\
\hline V1143 Ori & $B$ & 67 & 17.49 & 0.25 & 14.35 \\
& $V$ & 52 & 16.71 & 0.44 & 13.76 \\
& $R$ & 47 & 15.40 & 0.41 & 13.10 \\
& $I$ & 81 & 14.18 & 0.15 & 13.31 \\
\hline NY Ori & $B$ & 180 & 10.83 & 0.10 & 10.56 \\
& $V$ & 93 & 10.45 & 0.04 & 10.35 \\
& $R$ & 65 & 10.09 & 0.11 & 9.66 \\
& $I$ & 102 & 9.56 & 0.14 & 9.20 \\
\hline
\end{tabular}

Notes. For each source and each band, we list the number of observations (Col. 3), the median, which basically indicates the magnitude in quiescence (Col. 4), the standard deviation data point distribution (Col. 5), and the magnitude corresponding to the peak brightness (Col. 6)

sources V1118 Ori, V1143 Ori, and NY Ori are located. Observations of this region were repeatedly carried out at Asiago over a timespan of about 40 years since 1958 and the three sources were acquired on the same plates.
We provide one of the best-sampled photometric dataset ever obtained of the quiescent phase of the three targets. V1118 and V1143 present, in quiescence, a level of modest variability (0.2-0.4 mag) that is comparable with that of classical T Tauri stars.

For V1118 Ori, two already known outbursts are detected together with a newly discovered flare-up of $2.5 \mathrm{mag}$ ( $R$ band), which brightened up in only $40 \mathrm{~min}$ and lasted less than one month. Our data most likely ascertain the longest quiescence period known so far (about $15 \mathrm{yr}$ ): this result on the one hand does not support the existence of a recurrent period between the outbursts, on the other hand allows us to define a reliable value of the quiescence brightness. The colour analysis confirms the robustness of the plate photometry and rules out the extinction as the main origin of the brightness variations.

For V1143 Ori, two outbursts already mentioned in the past literature are found, but the complete set of colours are given here for the first time. This is relevant, since the colour analysis demonstrates as both outbursts, at variance with those of V1118, can be accounted for with pure extinction variations, putting some doubts on the EXor nature of V1143.

As for NY Ori, this target and the close source V566 Ori cannot be resolved in our plates, so that the given photometry refers to the combined pair. Nevertheless, as the two objects have a similar brightness in the I band, we can conclude that the EXor 
R. Jurdana-Šepić et al.: Past history of EXors
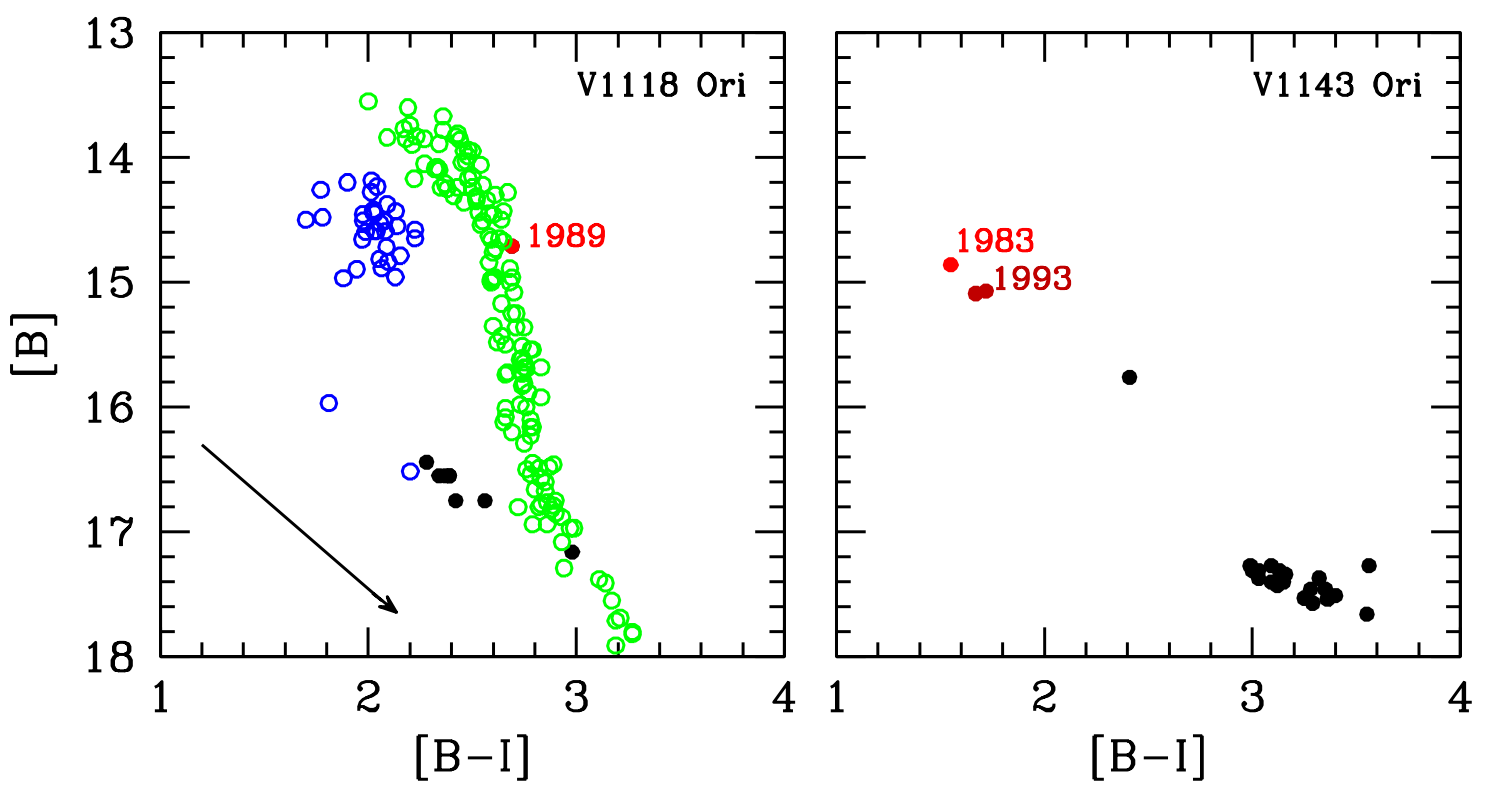

Fig. 4. $B$ vs. $[B-I]$ colour-magnitude plot of V1118 (left) and V1143 Ori (right). In the left panel open circles (in blue) refer to our data still unpublished (Giannini et al., in prep.). Solid circles (black and red) refer to the present data. Green circles are given for comparison purposes and refer to a recent outburst of V1118 monitored by Audard et al. (2005). In the lower-left corner the arrow indicates an extinction of $A_{\mathrm{V}}=1$ mag, according to the law by Rieke \& Lebofsky (1985).

source did not undergo any major outburst ( $>2$ mag) during the 40 yr monitoring.

Acknowledgements. R.J.S. thanks the INAF Astronomical Observatory of Padova for the hospitality during the stay in Asiago and for permission to use the historical plate archive of the Asiago Observatory. This work was supported in part by the Croatian Science Foundation under the project 6212 Solar and Stellar Variability and by the University of Rijeka under the project number 13.12.1.3.03.

\section{References}

Adams, F. C., \& Lin, D. N. C. 1993, Protostars and Planets III, 721

Antoniucci, S., Arkharov, A. A., Di Paola, A., et al. 2013, Protostars and Planets VI Posters, 2

Antoniucci, S., Arkharov, A. A., Di Paola, A., et al. 2014, A\&A, 565, L7

Audard, M., Güdel, M., Skinner, S. L., et al. 2005, ApJ, 635, L81

Audard, M., Stringfellow, G. S., Güdel, M., et al. 2010, A\&A, 511, A63

Audard, M., Ábrahám, P., Dunham, M. M., et al. 2014, Protostars and Planets VI, 387

Bell, K.R., \& Lin, D.N.C. 1994, ApJ, 427, 987

Bonnell, L., \& Bastien, P. 1992, ApJ 401, L31

D'Alessio, F., Di Cianno, A., Di Paola, A., et al. 2000, Proc. SPIE, 4008, 748

D’Angelo, C. R., \& Spruit, H. C. 2010, MNRAS, 406, 1208

García García, J., \& Parsamian, E.S. 2000, IBVS, 4925

Giannini, T., Lorenzetti, D., Antoniucci, S., et al. 2016, ApJ, 819, L5

Hartmann, L., \& Kenyon, S. 1985, ApJ, 299, 462

Henden, A., \& Munari, U. 2014, Contributions of the Astronomical Observatory Skalnate Pleso, 43, 518
Henden, A. A., Levine, S. E., Terrell, D., Smith, T. C., \& Welch, D. 2012, J. Am. Assoc. Variable Star Observers (JAAVSO), 40, 430

Herbig, G.H. 1989, in Proc. of the ESO Workshop on Low Mass Star Formation and Pre-Main Sequence Objects, ed. B. Reipurth, 233

Herbig, G. H. 2008, AJ, 135, 637

Jurdana-Šepić, R., \& Munari, U. 2016, New A., 43, 87

Kóspál, Á., Ábrahám, P., Acosta-Pulido, J. A., et al. 2011, A\&A, 527, A133

Lodato, G., \& Clarke, C.J. 2004, MNRAS, 353, 841

Lorenzetti, D., Giannini, T., Calzoletti, L., et al. 2006, A\&A, 453, 579

Lorenzetti, D., Giannini, T., Larionov, V. M., et al. 2007, ApJ, 665, 1182

Lorenzetti, D., Larionov, V. M., Giannini, T., et al. 2009, ApJ, 693, 1056

Lorenzetti, D., Antoniucci, S., Giannini, T., et al. 2012, ApJ, 749, 188

Lorenzetti, D., Antoniucci, S., Giannini, T., et al. 2015, ApJ, 802, 24

Mampaso, A., \& Parsamian, E.S. 1995, IBVS, 4269

Moro, D., \& Munari, U. 2000, A\&AS, 147, 361

Munari U. 2012, JAAVSO, 40, 582

Munari, U. 2014, in Astroplate 2014, eds. L. Misková, \& S. Vítek (Prague: The Institute of Chemical Technology)

Munari, U., \& Dallaporta, S. 2014, New A., 27, 25

Munari, U., Henden, A., Frigo, A., et al. 2014, AJ, 148, 81

Parsamian, E. S., \& Gasparian, K. G. 1987, Astrophysics, 27, 598

Parsamian, E. S., Ibragimov, M. A., Oganian, G. B., \& Gasparian, K. G. 1993, Astrophysics 36, 23

Paul, C., Kroll, P., \& Lehmann, T. 1995, Proc. IAU Colloq., 151, Sonneberg, 5-9 Dec., eds. J. Greiner, W. Hilmar. Duerbeck, \& E. Roald E. Gershberg, Lecture Notes in Physics, 454, 229

Rieke, G. H., \& Lebofsky, M. J. 1985, ApJ, 288, 618

Shu, F., Najita, J., Ostriker, E., et al. 1994, ApJ, 429, 781

Sicilia-Aguilar, A., Kóspál, Á., Setiawan, J., et al. 2012, A\&A, 544, A93

Xiao, L., Kroll, P., \& Henden, A.A. 2010, AJ, 139, 1527 